\title{
Un algorithme glouton pour le positionnement de capteurs qualité sur un grand réseau de distribution d'eau
}

\section{A greedy algorithm for quality sensor placement on a large-scale water distribution network}

\author{
N. CHEIFETZ' ${ }^{1}$ A.-C. SANDRAZ', C. FELIERS ${ }^{1}$, \\ D. GILBERT ${ }^{2}$, O. PILLER ${ }^{2}$, V. HEIM ${ }^{3}$
}

Mots-clés : placement/localisation de capteurs, qualité de l'eau, système d'alerte précoce de contaminations, algorithme d'optimisation, réseaux d'alimentation en eau potable (AEP), Syndicat des Eaux d'lle de France (SEDIF)

Keywords : sensor placement problem (SPP), water quality, contaminant warning system (CWS), early warning detection system (EWDS), optimization algorithm, drinking water distribution systems (DWDS), Syndicat des Eaux d'lle de France (SEDIF)

\begin{abstract}
Résumé
L'exploitation d'un système moderne d'alimentation en eau potable nécessite une surveillance permanente de la qualité de l'eau au sein des usines de production et sur le réseau de distribution, face à des risques de contamination accidentelle ou terroriste. Cette surveillance s'appuie usuellement sur un ensemble de capteurs déployés en des points stratégiques du réseau afin de minimiser l'impact de toute contamination. La localisation de capteurs consiste à déterminer des points du réseau avec la meilleure protection pour un nombre limité de capteurs ce qui représente un problème complexe en théorie et très difficile à résoudre en pratique. Nous décrivons ici une méthodologie fondée sur un algorithme efficace de type glouton, élaborée dans le cadre d'un projet franco-allemand ${ }^{4}$. Cette approche utilise un grand nombre de contaminations, simulées par un logiciel de modélisation hydraulique, et sélectionne itérativement les meilleurs positions en fonction d'un critère fixé à optimiser. La méthode est évaluée pour le déploiement de capteurs multiparamètres mesurant chlore, température, pression et conductivité sur le réseau du $\mathrm{SEDIF}^{5}$, plus grand réseau français de distribution d'eau potable.
\end{abstract}

\begin{abstract}
Operating modern drinking water distribution systems requires the ability to monitor water quality within production plants and the distribution network in order to mitigate damaging events like accidental or malicious contaminations. The surveillance systems are usually based on a sensor network deployed at strategic locations to minimize the contamination impact on the population. Such problem consists in selecting sensing locations with the best protection with a given number of sensors which is complex in theory and very challenging in practice. We describe here a methodology using an efficient greedy-like algorithm, designed during a Franco-German project ${ }^{3}$. This approach uses an extensive number of contaminations simulated by hydraulic modeling software and selects iteratively the best positions according to a given criterion to optimize. The method is evaluated with the deployment of multiparameters quality sensors (chlorine, temperature, conductivity and pressure) on the SEDIF network, the largest drinking water distribution network in France.
\end{abstract}

\footnotetext{
${ }^{1}$ Veolia Eau d'lle de France, Le Vermont, 28, Boulevard de Pesaro, F-92751 Nanterre, France. nicolas.cheifetz@veolia.com, anne-claire.sandraz@veolia.com, cedric.feliers@veolia.com

${ }^{2}$ IRSTEA, groupement de Bordeaux, UR REBX, 50 avenue de Verdun, F-33612 CESTAS Cedex, France. denis.gilbert@irstea.fr, olivier.piller@irstea.fr

${ }^{3}$ Syndicat des Eaux d'lle de France (SEDIF), 120 Boulevard Saint-Germain, F-75006 Paris, France. v.heim@sedif.com

${ }^{4}$ Projet SMaRT-Online wDN (2012-2015). http://www.smart-onlinewdn.eu/

${ }^{5}$ Syndicat des Eaux d'lle de France (SEDIF). http://www.sedif.com/
} 


\section{Introduction}

Les réseaux d'alimentation en eau potable sont depuis toujours des systèmes vulnérables et ont par le passé aussi bien été utilisés en tant qu'armes d'actions militaires étatiques ou cibles d'actes terroristes. Durant le siège d'Uxellodunum (Gaules) en 51 av. J.-C. lancé par Jules César, les forces romaines parviennent à détourner la source qui alimentait en eau la citadelle et obtiennent ainsi la capitulation des gaulois. Le lecteur intéressé par une chronologie des conflits liés à l'eau pourra se référer à la liste dressée par (Gleick \& Heberger, 2014). Suite aux événements survenus le 11 septembre 2001 aux Etats-Unis d'Amérique, l'association américaine analogue de l'ASTEE préconise notamment le développement de technologies peu onéreuses en terme d'infrastructure et automatique en terme de traitement de l'information, comme les "systèmes d'alertes " pour la surveillance des systèmes de distribution d'eau potable ((AWWA), 2005). La détection de contaminations accidentelles ou terroristes est permise par un ensemble de capteurs déployés en des points stratégiques du réseau afin de minimiser l'impact de toute contamination. Le positionnement « optimal » d'un ensemble de capteurs sur un réseau de distribution d'eau potable est un problème d'optimisation combinatoire NP-difficile (Krause, 2008; Xu, Lu, Huang, Xiao, \& Wang, 2013) ce qui signifie que la solution optimale à ce problème ne pourra pas être trouvée efficacement par tout algorithme actuel. De nombreuses contributions ont été proposées pour résoudre ce problème depuis la dernière décennie et on cite en particulier un challenge scientifique comparant quatorze méthodologies à partir de deux réseaux réalistes (Ostfeld, et al., 2008) et deux états de l'art regroupant à eux seuls près de cent cinquante articles (Hart \& Murray, 2010; Rathia \& Gupta, 2014). Malgré une forte activité scientifique sur ce sujet, il n'existe pas aujourd'hui de consensus clairement établi à propos d'un algorithme à utiliser ou d'une fonction objectif à optimiser.

Dans cet article, on s'intéresse au problème du placement de capteurs pour la surveillance de la qualité d'eau en réseau. D'autres stratégies de placement peuvent être employées à des fins de détection de fuites (Sarrate, Blesa, Nejjari, \& Quevedo, 2014) ou encore de calage de modèles hydrauliques (Kapelan, Savic, \& Walters, 2003). Notre objectif est de placer un nombre fixé de capteurs en des points stratégiques du réseau afin de minimiser l'impact sur les usagers de tout événement de contamination qu'il soit accidentel ou intentionnel. La stratégie proposée doit idéalement être en mesure de déclencher une alarme aussitôt qu'une substance contaminante potentiellement dangereuse pénètre le système de distribution et cela avant que tout consommateur soit atteint. Cette méthodologie globale doit être à la fois spécifique au réseau étudié pour l'utilisation de connaissances expertes et suffisamment flexible pour tenir compte des incertitudes liées à la nature, au temps et à la durée d'injection d'un contaminant. Dans le cadre du projet SMaRT-Online WDN ${ }^{4}$ financé par les agences nationales de recherche française et allemande (respectivement ANR-11SECU-006 et BMBF-13N12180), Veolia Eau d'lle de France a participé à la conception et évalué la performance d'une méthode de déploiement de capteurs qualité sur le réseau du Syndicat des Eaux d'lle de France (SEDIF) ${ }^{5}$ présenté dans cet article. Ce réseau d'alimentation en eau potable est le plus grand réseau français de distribution et parmi les plus grands d'Europe. Le syndicat, qui regroupe 149 communes autour de Paris, distribue chaque jour à plus de quatre millions d'habitants près de $750000 \mathrm{~m}^{3}$ d'eau potable à partir de trois usines alimentées par la Seine, la Marne et l'Oise. Le réseau comptabilise plus de $8000 \mathrm{~km}$ de canalisations et peut être modélisé à partir de onze modèles hydrauliques de taille variable, illustrés par la figure 1 ; un déploiement sera réalisé sur chacun de ces modèles indépendamment. La grande taille d'un réseau comme celui étudié exclut le recours à des méthodes empiriques comme une évaluation exhaustive (trop coûteuse en temps de calcul) ou encore à un placement aléatoire (trop de solutions possibles pour être efficace).

La première section décrit la stratégie proposée ainsi que les choix méthodologiques pour le déploiement de capteurs qualité. La section 2 présente les résultats obtenus pour le placement de capteurs sur le réseau du SEDIF, et à titre d'illustration sur le réseau de l'Eurométropole de Strasbourg (EMS) également dans le cadre du projet SMaRT Online. Enfin, on termine l'article par quelques conclusions et perspectives. 
(a)

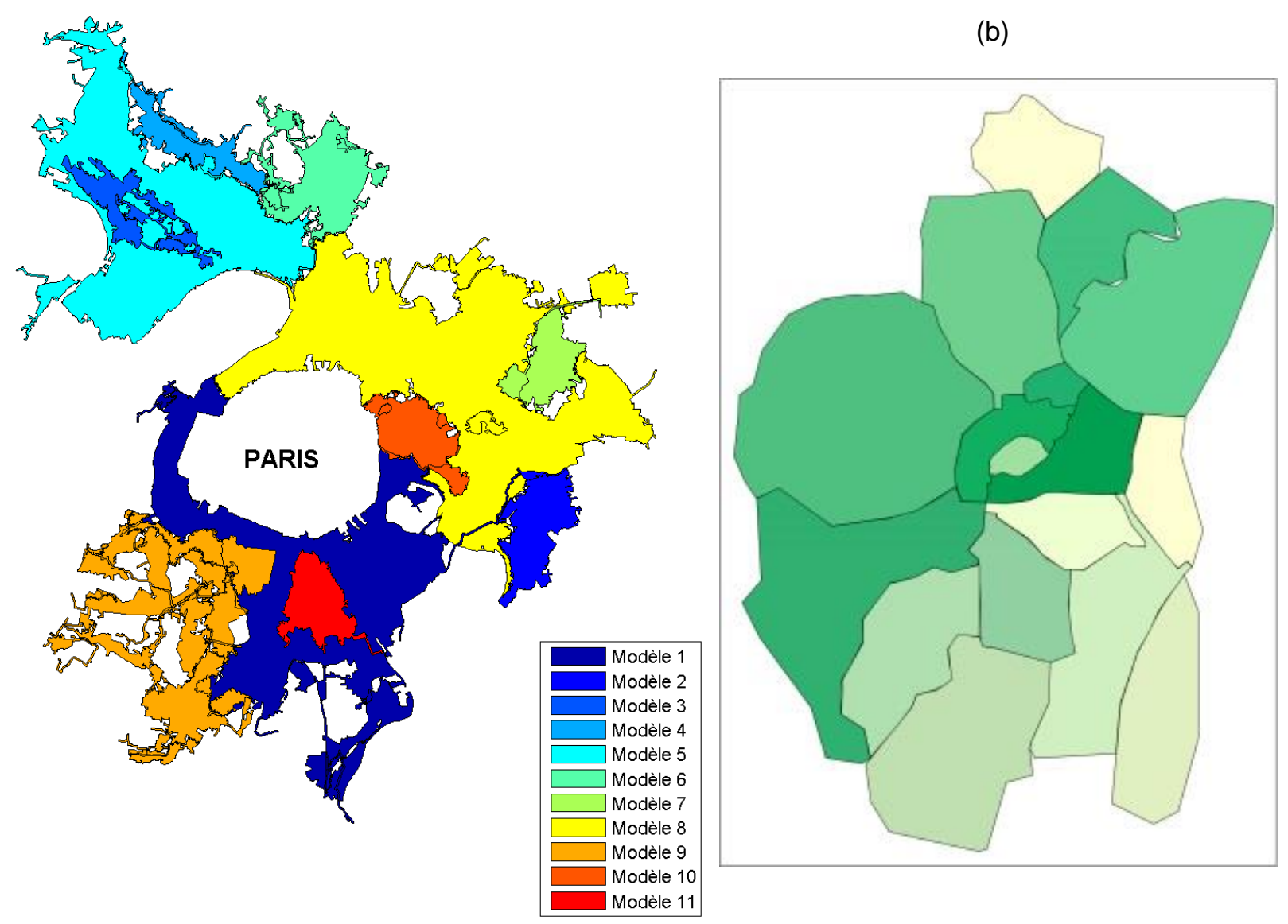

Figure 1 : Territoires des deux réseaux de distribution en eau potable.

Territoire du SEDIF, autour de Paris, et sa décomposition en onze modèles hydrauliques (a).

Territoire du réseau de l'Eurométropole de Strasbourg (EMS) avec 14 secteurs (b).

\section{Déploiement de capteurs mesurant la qualité d'eau}

De nombreuses mesures de performance ont été proposées pour quantifier l'efficacité des solutions de placement. Certains critères ont pour objet de faciliter la procédure de décontamination comme la minimisation du volume d'eau consommé ou celle de la surface des canalisations avant détection par un capteur. La minimisation de l'impact de contaminations sur les usagers est un objectif global usuellement accepté par la communauté scientifique (Hart \& Murray, 2010) et on illustre les solutions obtenues après minimisation du ratio de population exposée dans la sous-section 2.2. II est à noter que la méthodologie proposée offre la possibilité d'optimiser différents critères dans la mesure où ils peuvent être formulés après l'occurrence de multiples incidents simulés de contamination sur le réseau de distribution.

\subsection{Caractérisation de l'impact de contaminations}

L'impact de contaminations est quantifié à partir d'un grand nombre d'injections de contaminant simulées par des outils de modélisation hydraulique, comme par exemple EPANET (Rossman, 2000) ou encore Porteau ${ }^{6}$. Dans ce type de simulations, on injecte une substance à partir d'un nœud du modèle hydraulique puis la contamination est détectée lorsque la dose de la substance est supérieure

\footnotetext{
${ }^{6}$ Porteau est un logiciel libre pour la modélisation des réseaux de distribution ou de transport d'eau sous pression. http://porteau.irstea.fr/
} 
à un seuil fixé au niveau d'un capteur. On s'intéresse à l'impact moyen d'un ensemble de contaminations plutôt que l'impact du pire incident, respectivement dans des cas de contaminations accidentelles ou intentionnelles. En effet, dans notre étude et le plus souvent, la spécification des contaminations futures est inconnue et on suppose que les formes de contaminations peuvent changer au cours du temps. On peut notamment montrer qu'un réseau de capteurs établi à partir de contaminations accidentelles surpasse le plus souvent tout autre réseau de capteurs obtenu dans le pire des cas, lorsque les conditions de contamination changent (Davis, Janke, \& Phillips, 2013).

Par conséquent, l'ensemble des simulations de contamination constitue ici l'information clé pour l'optimisation d'un placement de capteurs. Des scénarios de contamination sont générés aléatoirement de manière à prendre en compte l'incertitude liée à la nature, au temps et à la durée de chaque contamination potentielle, en suivant les spécifications suivantes :

- Nombre de simulations : à fixer en fonction de la convergence du critère à optimiser (on choisit aléatoirement un ensemble de nœuds du modèle hydraulique pour chaque contamination, de telle sorte qu'environ un tiers des nœuds sont contaminés à l'issue de toutes les simulations) ;

- Durée de chaque simulation : dépend du temps de séjour dans le réseau (a priori quelques journées suffisent pour que la solution ne dépende plus des condition initiales);

- Nature de la substance injectée : un contaminant conservatif a été choisi en raison de son impact fort en diffusion ; il n'y a pas de dilution hormis celle induite par le mélange au niveau des nœuds ;

- Temps de début et durée d'injection : la substance est injectée aléatoirement entre 0h et $24 \mathrm{~h}$ pendant une durée choisie entre $0 \mathrm{~h}$ et $6 \mathrm{~h}$ aléatoirement (distribution de probabilité uniforme) ;

- Localisation de l'injection: aléatoire parmi les nœuds du modèle (distribution de probabilité uniforme) ;

- Détection d'une contamination : la concentration de la substance injectée est supérieure à un seuil fixé dont la valeur dépend de la concentration initialement injectée.

Dans cette étude, les contaminations ont été simulées à partir du logiciel Synergi Water ${ }^{\circledR}$ (DNV GL) pour le réseau du SEDIF, et à partir du logiciel Porteau pour le réseau de Strasbourg.

Après la simulation d'un grand nombre d'injections, toute l'information relative aux contaminations est agrégée dans une unique matrice de contaminations par modèle hydraulique. On peut représenter cet objet sous la forme d'une matrice tridimensionnelle, illustrée par la figure 2. La dimension des caractéristiques décrit toutes les variables nécessaires pour le calcul des critères de performance du placement : le temps depuis l'injection d'une substance, la somme cumulée des branchements sur les nœuds exposés, etc. II est à noter que si une contamination n'est pas détectée, chaque critère est tout de même calculé à la fin de la simulation de manière à pénaliser les incidents non détectés. Les dimensions des contaminations et des nœuds du modèle listent respectivement chaque injection de contaminant et chaque nœud du modèle hydraulique. On remarque que cette matrice est parcimonieuse (beaucoup de valeurs nulles) à cause du nombre limité de nœuds exposés pour chaque contamination simulée ; autrement dit, il existe un nombre souvent important de nœuds qu'il est inutile d'évaluer à chaque contamination donnée.

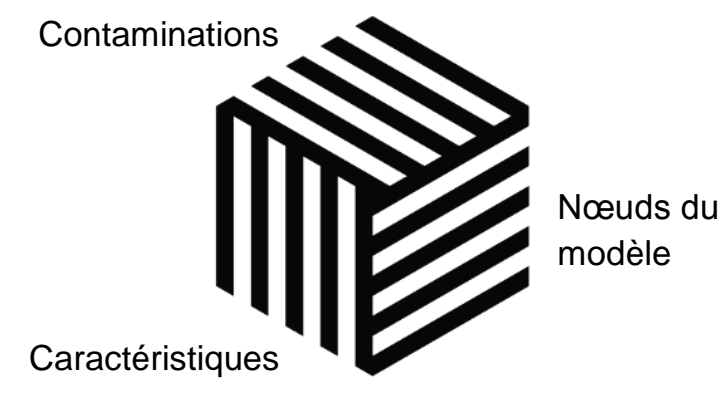

Figure 2 : Matrice des contaminations construite pour chaque modèle hydraulique. 


\subsection{Placement incrémental de capteurs}

La méthodologie proposée dans cet article s'appuie sur des matrices de contamination pour caractériser l'impact de contaminations aléatoires. Cette méthode permet aussi de prendre en compte facilement de nombreuses connaissances expertes suivant deux motivations principales.

Les premiers a priori concernent des connaissances expertes sur les capteurs qualité ${ }^{7}$ :

- Les capteurs sont positionnés sur des nœuds dont au moins une des canalisations adjacentes a un diamètre nominal ni trop grand ni trop petit ;

- La vitesse dans les canalisations adjacentes ne peut pas être trop faible de manière à ne pas sousestimer la concentration en chlore actif ;

- Pas d'inversion du sens d'écoulement au niveau des sondes ;

Les seconds a priori sont visent à accélérer l'algorithme d'optimisation :

- Les capteurs sont placés sur des nœuds spécifiques, appelés « super nœuds ". L'identification de ces nœuds s'appuie sur une méthodologie de décomposition de graphe proposée par (Deuerlein, 2008) : chacun des nœuds possède au moins deux voisins et n'appartient pas à des forêts dans le graphe. Par conséquent, le nombre de solutions à évaluer est significativement réduit ; - Les nœuds déjà équipés par des capteurs préexistants sont pris en compte mais ne sont évidemment pas évalués pour le placement potentiel de nouveaux capteurs. Dans une application réelle, la stratégie de placement peut être régulièrement utilisée pour le renouvellement du réseau de capteurs ou la mise en place d'un nouveau déploiement.

La méthode d'optimisation proposée dans cet article est un algorithme glouton ce qui signifie que tout nouveau capteur est placé à chaque itération de l'algorithme, et cela sur la base des capteurs déjà positionnés précédemment. Plus précisément, on choisit de placer un capteur sur le nœud qui améliore le plus le critère optimisé (maximisé ou minimisé selon le cas) à chaque itération. II s'agit d'une méthode heuristique qui conduit à l'obtention d'une solution sous-optimale mais de telles approches incrémentales sont reconnues pour leur rapidité d'exécution et permettent d'obtenir des solutions proches de l'optimale pour ce type de problèmes. En effet, la fonction combinatoire à optimiser est sous-modulaire (Krause, 2008; Xu, Lu, Huang, Xiao, \& Wang, 2013) et celle-ci est améliorée à chaque ajout de capteur (par construction). Intuitivement, l'ajout d'un capteur après le déploiement d'un grand réseau de capteurs apporte moins d'information que l'ajout d'un capteur à un faible déploiement. Ce concept est formalisé par le concept combinatoire de sous-modularité.

On synthétise la méthodologie de positionnement proposée par trois étapes principales successives illustrées par la figure 3. La première étape concerne la génération de scénarios aléatoires de contamination et leurs simulations via un outil de modélisation hydraulique. Sur la base de ces simulations et d'informations a priori, on construit une matrice de contaminations pour chaque modèle hydraulique ce qui correspond à la seconde étape. Enfin, l'algorithme glouton est alimenté par les matrices de contaminations et des connaissances expertes de manière à positionner un ensemble de capteurs et les évaluer en fonction de différents critères.

\footnotetext{
${ }^{7}$ Sondes multiparamètres Kapta ${ }^{\text {TM }} 3000$ commercialisés par (ENDETEC, 2012) et développés dans le cadre du projet européen SecurEau. http://www.secureau.eu/
} 


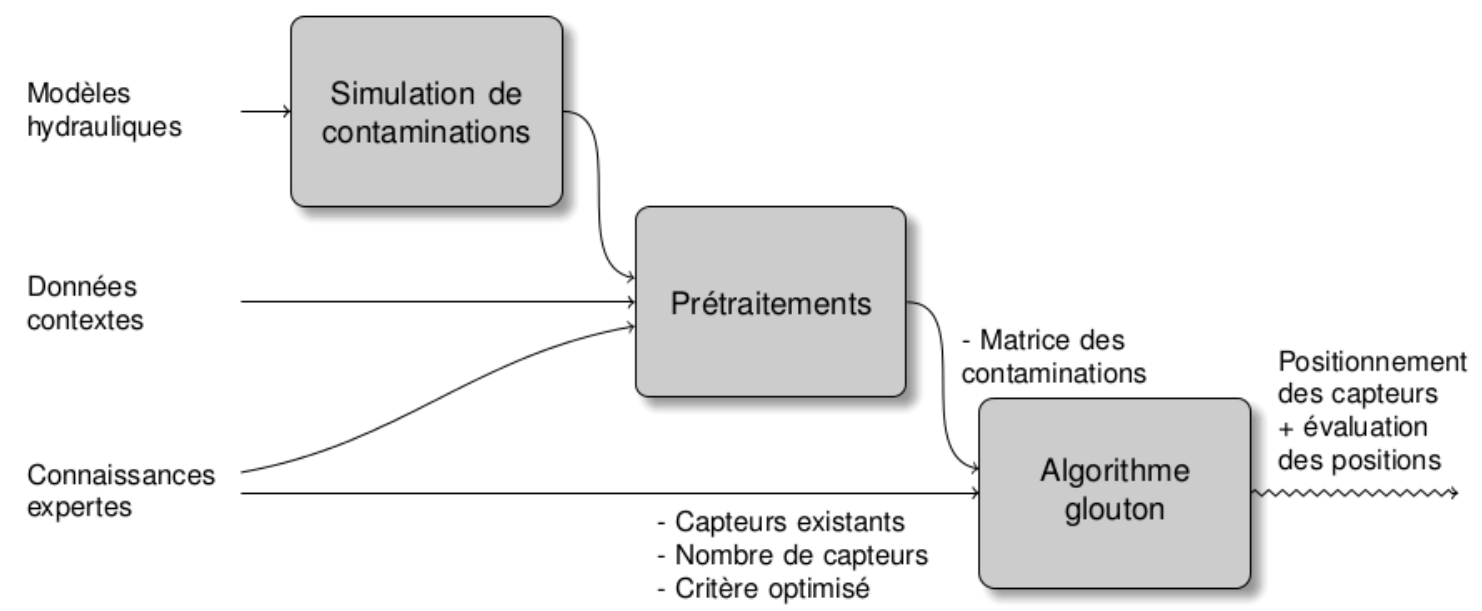

Figure 3 : Méthodologie globale de l'approche proposée pour le placement incrémental de capteurs.

\section{Evaluation de la stratégie de déploiement}

\subsection{Description du cas d'étude}

La stratégie de placement est appliquée sur le réseau du SEDIF pour le positionnement d'environ deux cents capteurs déployés sur les onze modèles hydrauliques illustrés par la figure 1. Dans le cadre de nos travaux, un premier déploiement d'une centaine de capteurs avait déjà été réalisé sur la base de connaissances expertes du réseau et un modèle hydraulique avait déjà été équipé pour les besoins du projet SMaRT-Online ${ }^{\text {WDN }}$. L'objectif global est par conséquent de placer une centaine de capteurs parmi plus de 100000 nœuds (répartis sur dix modèles hydrauliques) ce qui représente une combinaison de $10^{2}$ parmi $10^{5}$ soit environ $10^{342}$ solutions potentielles. Le tableau I décrit le réseau de capteurs déployé sur le réseau du SEDIF. Le nombre de capteurs a été choisi au prorata du nombre de nœuds dans chaque modèle hydraulique (voir la sous-section 1.1) ; on remarque notamment que les modèles 1, 5 , 8 et 9 sont les plus grands modèles du réseau et nécessitent ainsi l'installation d'un plus grand nombre de capteurs. Le modèle hydraulique représentant le réseau de Strasbourg contient environ 13500 nœuds ce qui implique un placement de 27 capteurs pour rester dans le même ordre de grandeur que le ratio utilité pour le placement du SEDIF.

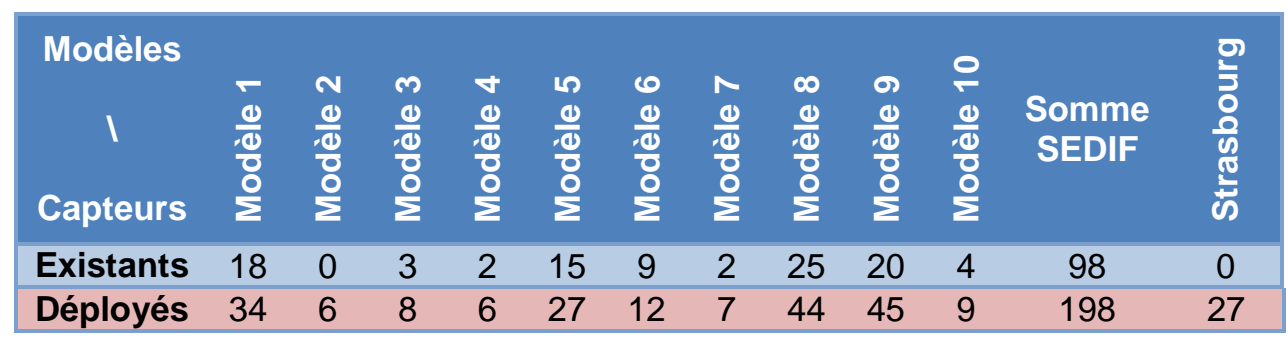

Tableau I : Le nombre de capteurs déployés par modèle hydraulique sur le réseau du SEDIF, ainsi que celui de Strasbourg.

Dans notre analyse, on quantifie l'impact des contaminations sur les usagers à partir des quatre critères de performance suivants:

- Ratio de la population exposée (PE) : le nombre de branchements exposés aux contaminations avant détection par un capteur, normalisé par le nombre de branchements dans chacun des modèles hydrauliques ;

- Ratio de la population vulnérable exposée (PVE) : la somme des facteurs de vulnérabilité au niveaux 
des nœuds exposés aux contaminations avant détection par un capteur. Un facteur de vulnérabilité est attachée à chaque nœud et celle-ci est attribuée à dire d'expert selon la connaissance disponible des consommateurs vulnérables (écoles, hôpitaux, dialysés,...) ;

- Délais à la détection (DT) : durée entre le temps d'injection d'une substance et la détection de la contamination par un capteur ;

- Nombre de détection (ND) : Nombre de fois où l'intrusion a été détectée par un capteur.

On rappelle que l'on s'intéresse à l'impact moyen des contaminations ce qui signifie que ces critères sont moyennés sur l'ensemble des contaminations simulées.

Par ailleurs, ces quatre critères supposent que les capteurs qualité sont «parfaits » dans le sens où ils sont toujours précis et fiables (ni faux positifs ni faux négatifs et aucune panne). Ces capteurs parviennent toujours à détecter une contamination qui passe par l'un d'eux et l'information est transmise instantanément. Ces hypothèses sont appelées en anglais "perfect sensors » et "perfect response "(Clark, Hakim, \& Ostfeld, 2011). Une contamination est ainsi détectée lorsque la concentration de contaminant en ce nœud est non nulle, ce qui nécessite en pratique de fixer un seuil de concentration minimale non seulement à cause de la précision machine mais aussi car cette hypothèse de modélisation est plus réaliste dans la réalisation du système de surveillance (Hart \& Murray, 2010). Les critères qui en découlent permettent d'estimer en quelque sorte une borne inférieure (cas de minimisation) de la performance d'un ensemble de capteurs.

\subsection{Résultats obtenus}

Cette sous-partie présente les résultats obtenus pour le positionnement d'un ensemble de capteurs sur chaque modèle hydraulique par minimisation du ratio moyen de la population exposée (PE). On illustre les quatre critères de performance normalisés avec les valeurs obtenues dans la situation où aucun capteur n'est positionné sur le réseau. La figure 4 représente la contribution de chaque réseau de capteurs sous la forme d'histogrammes qu'il convient de maximiser. Avec le placement d'environ deux cents capteurs sur les dix modèles hydrauliques du SEDIF, le critère PE optimisé et normalisé est entre $75 \%$ et $90 \%$ environ pour chaque modèle ; la performance de ce critère à partir des réseaux de capteurs existants est entre $0 \%$ (pas de capteurs) et 70\%. II est à noter que le placement d'un grand nombre de capteurs n'implique pas nécessairement une meilleure efficacité en comparaison avec des modèles hydrauliques moins fournis, comme le modèle 4 par exemple. Aussi, on remarque que les critères $P E$ et PVE présentent des résultats similaires ce qui s'explique par leurs formulations analogues. Enfin, nous avons pu observer que le critère ND est un critère différent des autres dans le sens où l'objectif est de maximiser les détections. Autrement dit, ce critère propose des localisations de capteurs vers les extrémités du réseau afin de détecter un maximum d'intrusions, ce qui apparait comme une approche non intuitive. Les critères obtenus sur le réseau de Strasbourg sont similaires et semblent parfois meilleurs que ceux des modèles du SEDIF ; cela s'explique par une topologie différente du réseau hydraulique mais également par le fait que tous les capteurs placés sur le réseau de Strasbourg sont optimisés (sans capteurs existants).

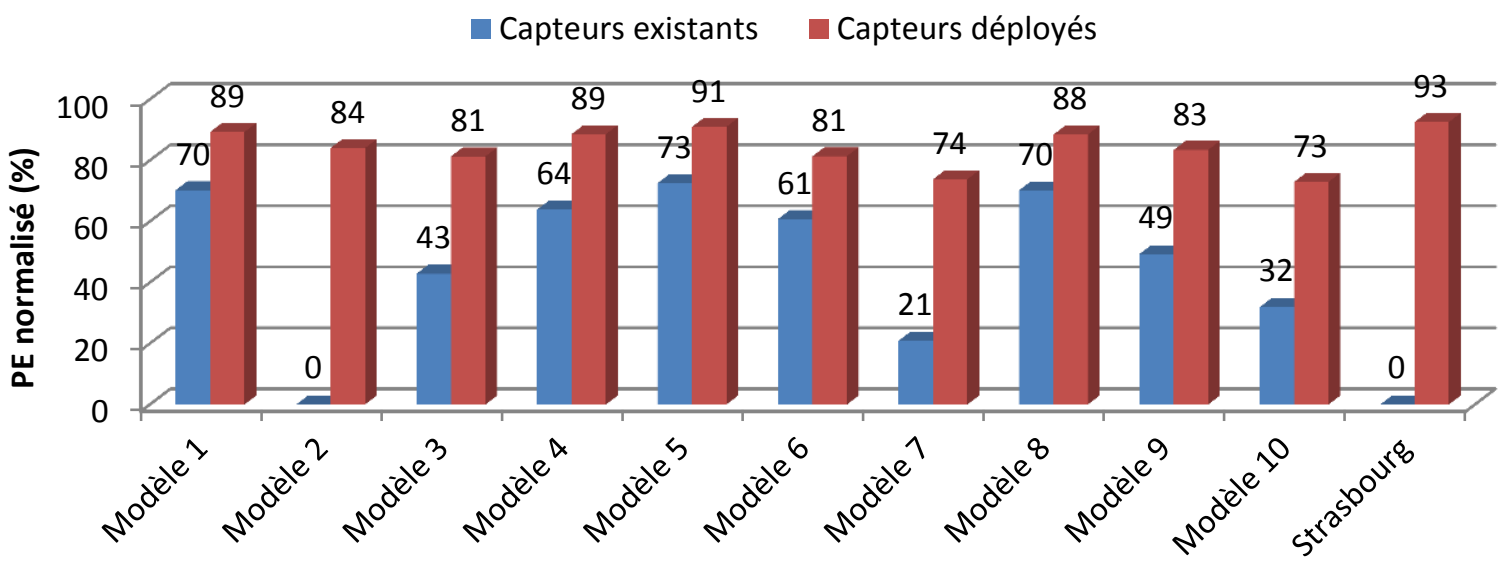



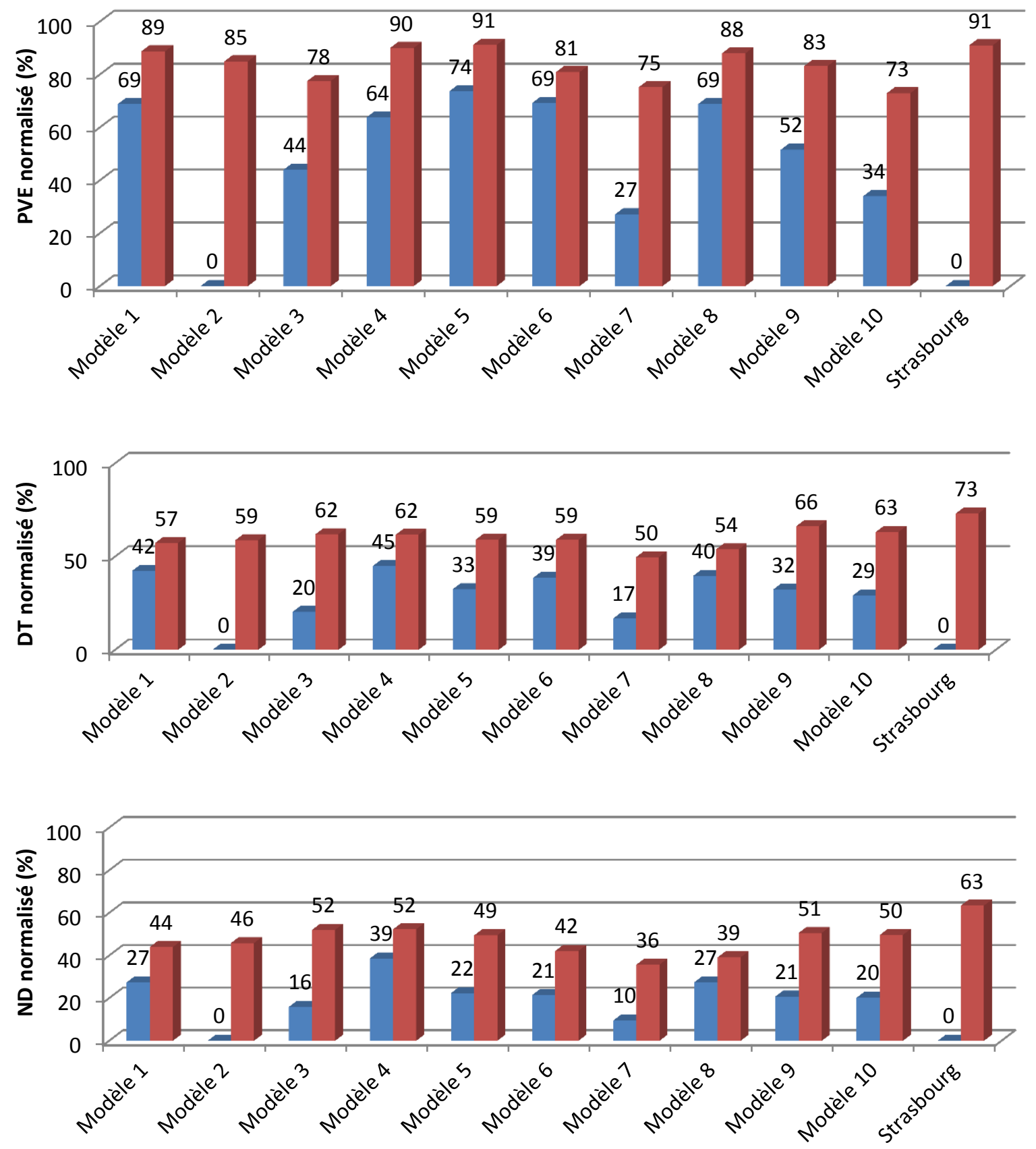

Figure 4 : Evaluation du déploiement des capteurs à partir de quatre critères normalisés.

La signification de chaque critère est PE : le ratio moyen de la population exposée,

PVE : le ratio moyen de la population vulnérable exposée,

DT : le délais moyen à la détection, et

ND : la probabilité de détection.

\section{Conclusion et perspectives}

Dans cet article, nous avons présenté une méthodologie flexible pour le déploiement de capteurs qualité sur des réseaux d'alimentation en eau potable. Devant la difficulté d'un passage à l'échelle sur un grand réseau de distribution comme celui du SEDIF étalé sur plus de $8000 \mathrm{~km}$ de conduites, un algorithme incrémental de type glouton a été proposé. Les propriétés de sous-modularité de cet algorithme s'avèrent particulièrement bien adaptées au problème adressé. L'efficacité de cette approche nous a permis de positionner une centaine de nouveaux capteurs au sein du réseau du SEDIF sur la base d'un réseau de capteurs précédemment déployé. Le placement obtenu a été 
évalué en fonction de quatre critères de performance. A titre d'illustration, les résultats du placement de 27 sondes ont été présentés sur le réseau de l'Eurométropole de Strasbourg. En termes de perspectives, l'algorithme glouton pourra être comparé à d'autres approches heuristiques ou d'autres méthodes de résolution exacte comme par exemple un programme mathématique mixte. A notre connaissance, ce problème de placement est intrinsèquement multi-objectif et nécessite de réaliser un compromis entre un parcours relativement coûteux du front de Pareto et un faible temps de calcul.

\section{Bibliographie}

(AWWA), A. W. (2005). CONTAMINATION WARNING SYSTEMS FOR WATER: An Approach for Providing Actionable Information to Decision-Makers. Tech. rep.

Clark, R., Hakim, S., \& Ostfeld, A. (2011). Handbook of Water and Wastewater Systems Protection. Springer.

Davis, M., Janke, R., \& Phillips, C. (2013). Robustness of Designs for Drinking Water Contamination Warning Systems under Uncertain Conditions. Journal of Water Resources Planning and Management.

Deuerlein, J. W. (2008). Decomposition model of a general water supply network graph. Journal of Hydraulic Engineering, 134(6), 822-832.

ENDETEC. (2012, dec). Kapta 3000 AC4 - Sensor Module Application Note. Tech. rep., Veolia Water Solutions \& Technologies.

Gleick, P. H., \& Heberger, M. (2014). Water conflict chronology. Dans The world's water (pp. 173219). Springer.

Hart, W. E., \& Murray, R. (2010). Review of sensor placement strategies for contamination warning systems in drinking water distribution systems. Journal of Water Resources Planning and Management, 136(6), 611-619.

Kapelan, Z. S., Savic, D. A., \& Walters, G. A. (2003). Multiobjective sampling design for water distribution model calibration. Journal of Water Resources Planning and Management, 129(6), 466-479.

Krause, A. (2008). Optimizing sensing: theory and applications. Thèse de Doctorat, Carnegie Mellon.

Ostfeld, A., Uber, J., Salomons, E., Berry, J., Hart, W., Phillips, C., . . Walski, T. (2008). The Battle of the Water Sensor Networks (BWSN): A Design Challenge for Engineers and Algorithms. Journal of Water Resources Planning and Management, 134(6), 556-568.

Rathia, S., \& Gupta, R. (2014). Sensor placement methods for contamination detection in water distribution networks: a review. 16th Conference on Water Distribution System Analysis, WDSA 2014.

Rossman, L. A. (2000). EPANET 2, Users Manual. Tech. rep., National Risk Management Research Laboratory, Office of Research and Development, U.S. Environmental Protection Agency.

Sarrate, R., Blesa, J., Nejjari, F., \& Quevedo, J. (2014). Sensor placement for leak detection and location in water distribution networks. Water Science and Technology: Water Supply.

Xu, X., Lu, Y., Huang, S., Xiao, Y., \& Wang, W. (2013). Incremental Sensor Placement Optimization on Water Network. Dans H. Blockeel, K. Kersting, S. Nijssen, \& F. Zelezny (Éds.), Machine Learning and Knowledge Discovery in Databases (Vol. 8190, pp. 467-482). Springer Berlin Heidelberg. 